$\Rightarrow$ IMMUNE TOLERANCE

\title{
Arming the front line with TLR9
}

Differential signalling from Toll-like receptor 9 (TLR9) on the apical and basolateral surfaces of intestinal epithelial cells (IECs) might provide one explanation for the normal immune tolerance to commensal bacteria in the gut, according to a study in Nature Cell Biology. IECs are highly polarized structures as a result of the markedly different environments that they interact with at the apical surface (the microflora of the intestinal lumen) and the basolateral surface (the lamina propria). Lee et al. investigated how such polarity affects the immune response to TLR ligands.

Polarized IECs secrete interleukin-8 (IL-8) into the basolateral environment after basolateral, but not apical, TLR9 stimulation with the ligand ISS-ODN (immunostimulatory sequence oligodeoxynucleotide). Consistent with this, ISS-ODN activated nuclear factor- $\kappa \mathrm{B}(\mathrm{NF}-\kappa \mathrm{B})$

from the basolateral surface only. The authors showed that TLR9 is expressed on both basolateral and apical surfaces of IECs (compared with its endosomal location in immune cells) and that TLR9 ligands can be taken up both apically and basolaterally. Therefore, the differential response to ISS-ODN must result from different signalling pathways in response to apical and basolateral TLR9 stimulation.

Whereas IKK (IאB kinase) activation and $\mathrm{I} \kappa \mathrm{B} \alpha$ (inhibitor of NF- $\mathrm{B} \mathrm{B}$ ) phosphorylation and ubiquitylation were similar in response to apical and basolateral TLR9 stimulation, I $\mathrm{B} \alpha$ was not degraded by the proteasome after apical TLR9 simulation. This stabilization of $\mathrm{I} \kappa \mathrm{B} \alpha$ prevented $\mathrm{NF}-\kappa \mathrm{B}$ activation in apically stimulated cells. The NF- $\kappa \mathrm{B} 1$ precursor p105 was also not degraded after apical TLR9 stimulation compared with basolateral stimulation. Because the ubiquitylation of both p 105 and $\mathrm{I} \kappa \mathrm{B} \alpha$ is mediated by the ubiquitin ligase $\beta$-TrCP, the authors suggest that there might be a general failure to degrade $\beta$-TrCPubiquitylated proteins after apical TLR9 stimulation. The authors propose that the inhibited NF- $\mathrm{\kappa B}$ response to apical TLR9 stimulation could induce a state of unresponsiveness to pro-inflammatory TLR signalling from both apical and basolateral IEC surfaces by increasing the NF- $\mathrm{KB}$ activation threshold. IECs that were apically pre-stimulated with ISSODN gradually lost the IL-8 response to basolateral TLR9 stimulation, as well as to apical TLR2 and basolateral TLR3 or TLR5 stimulation. Apical pre-stimulation with ISS-ODN resulted in the accumulation of ubiquitylated $\mathrm{I} \kappa \mathrm{B} \alpha$ and the failure to activate NF- $\kappa \mathrm{B}$ after secondary stimulation.

To assess the physiological relevance of this apical TLR9-mediated 'tolerance' in IECs, Lee et al. looked at the susceptibility of $T l r 9^{-/-}$mice to colonic inflammation induced by DSS (dextran-sulphate sodium salt). NF- $\kappa \mathrm{B}$ was activated at earlier time points after administration of DSS in Tlr $9^{-/-}$mice compared with wild-type mice; moreover, the Tlr $9^{-/-}$mice had larger areas of epithelial ulceration accompanied by inflammatory-cell infiltrates.

Therefore, the separation of apical TLR signals from basolateral TLR signals, owing to the polarized structure of IECs, could contribute to homeostasis in the intestinal tract.

Kirsty Minton

ORIGINAL RESEARCH PAPER Lee, J. et al.

Maintenance of colonic homeostasis by

distinctive apical TLR9 signalling in intestinal epithelial cells. Nature Cell Biol. 8, 1327-1336 (2006)

\section{RESEARCH HIGHLIGHTS ADVISORS}

CEZMI AKDIS Swiss Institute of Allergy and Asthma Research, Davos, Switzerland BRUCE BEUTLER

The Scripps Research Institute, La Jolla, USA PETER CRESSWEL

PETER CRESSWELL
Yale University, New Haven, USA
JAMES DI SANTO

Institut Pasteur, Paris, France GARY KORETZKY University of Pennsylvania, Philadelphia, USA CHARLES MACKAY

Garvan Institute of Medical Research, Sydney, Australia
CORNELIS MELIEF

Leiden University Medical Center, Leiden, The Netherlands MICHEL NUSSENZWEIG The Rockefeller University, New York, USA RICHARD RANSOHOFF

The Cleveland Clinic Foundation, Cleveland, USA
ALAN SHER

National Institute of Allergy and Infectious Diseases, Bethesda, USA ANDREAS STRASSER The Walter and Eliza Hall Institute of Medical Research, Melbourne, Australia
MEGAN SYKES

Harvard Medical School, Boston, USA ERIC VIVIER

Centre d'Immunologie de MarseilleLuminy, Marseille, France MATTHIAS VON HERRATH

La Jolla Institute for Allergy and Immunology, San Diego, USA. 